\title{
Structural studies of Helicase NS3 variants from Hepatitis $C$ virus genotype 3 in virological sustained responder and non-responder patients
}

\author{
Paola JS Provazzi ${ }^{1}$, Helen A Arcuri ${ }^{2}$, Isabel Maria VG de Carvalho-Mello ${ }^{3}$, João Renato R Pinho ${ }^{4}$, \\ Maurício L Nogueira ${ }^{5}$, Mário S Palma ${ }^{6}$, Paula Rahal ${ }^{1 *}$
}

\begin{abstract}
Background: About 130 million people are infected with the hepatitis C virus (HCV) worldwide, but effective treatment options are not yet available. One of the most promising targets for antiviral therapy is nonstructural protein 3 (NS3). To identify possible changes in the structure of NS3 associated with virological sustained response or non-response of patients, a model was constructed for each helicase NS3 protein coding sequence. From this, the goal was to verify the interaction between helicases variants and their ligands.

Findings: Evidence was found that the NS3 helicase portion of non-responder patients contained substitutions in its ATP and RNA binding sites. K210E substitution can cause an imbalance in the distribution of loads, leading to a decrease in the number of ligations between the essential amino acids required for the hydrolysis of ATP. W501R substitution causes an imbalance in the distribution of loads, leading and forcing the RNA to interact with the amino acid Thr269, but not preventing binding of ribavirin inhibitor.

Conclusions: Useful information is provided on the genetic profiling of the HCV genotype 3, specifically the coding region of the NS3 protein, improving our understanding of the viral genome and the regions of its protein catalytic site.
\end{abstract}

\section{Background}

Hepatitis $C$ virus $(\mathrm{HCV})$ is the main causative agent of non-A and non-B hepatitis. The clinical manifestations of infection include acute and chronic forms of hepatitis $\mathrm{C}$, liver cirrhosis and hepatocellular carcinoma [1]. The overall prevalence estimated of $\mathrm{HCV}$ infection is $2.2 \%$, which corresponds to 130 million $\mathrm{HCV}$-positive people in the world [2]. About 3 million people in Brazil are infected [3].

The HCV genome contains a positive single-stranded RNA of $\sim 9.6 \mathrm{~kb}$. It encodes a single precursor polyprotein containing $\sim 3000$ amino acids [4-6], which gives rise to all viral structural proteins (S) - core (protein C), envelope 1 (E1) and envelope 2 (E2) - and nonstructural proteins (NS), located in the following order: NS2, NS3, NS4A, NS4B, NS5A, and NS5B [7].

\footnotetext{
* Correspondence: Rahalp@yahoo.com.br

1 São Paulo State University - UNESP, Department of Biology, São José do Rio
} Preto/SP, CEP: 15054-000, Brazil
The NS3 protein of the hepatitis $\mathrm{C}$ virus ( $\mathrm{HCV}$ ) is a target for development of antiviral agents. It is a hydrophobic protein of $\sim 69 \mathrm{kDa}$, with its serine-protease function encoded in its $\mathrm{N}$-terminal portion accounting for one-third of the entire protein [7]. The C-terminal portion of the structural protein NS3 corresponds to the helicase domain, having NTPase and RNA helicase activities [8].

To identify possible changes in the structure of the NS3 protein associated with virologically sustained responder and non-responder patients, a model was constructed for each helicase NS3 protein coding sequence.

\section{Methods}

Population and samples

The study material consisted of the serum samples of the 16 patients infected with hepatitis $C$ virus genotype 3 . After confirming the positive diagnosis of infection, defined by positivity for the virus antibody by ELISA and qualitative PCR for RNA, the patients were given 24 weeks 
Table 1 Patients' characteristics

\begin{tabular}{|c|c|c|c|c|c|}
\hline & SEX & AGE (YEARS) & HCV GENOTYPE & SUBSTITUTION & RESPONSE \\
\hline RF006 & M & 39 & $3 a$ & & Non-responder \\
\hline RF007 & $\mathrm{F}$ & 28 & $3 e$ & K210E ATP binding site & Non-responder \\
\hline RF009 & M & 51 & $3 a$ & & Non-responder \\
\hline RF015 & M & 50 & $3 a$ & & Virological sustained responder \\
\hline RF018 & M & 49 & $3 a$ & & Virological sustained responder \\
\hline RF020 & M & 41 & $3 e$ & W501R RNA binding site & Non-responder \\
\hline RF059 & $\mathrm{F}$ & 54 & $3 a$ & F444S & Virological sustained responder \\
\hline RF060 & M & 48 & $3 a$ & & Non-responder \\
\hline RF061 & M & 38 & $3 e$ & & Virological sustained responder \\
\hline RF075 & $\mathrm{F}$ & 46 & $3 a$ & & Non-responder \\
\hline RF080 & M & 52 & $3 a$ & & Virological sustained responder \\
\hline RF081 & M & 58 & $3 a$ & & Virological sustained responder \\
\hline RF082 & $\mathrm{F}$ & 57 & $3 a$ & & Non-responder \\
\hline RF096 & M & 60 & $3 a$ & & Virological sustained responder \\
\hline RF115 & M & 41 & $3 a$ & & Non-responder \\
\hline RF145 & M & 51 & $3 a$ & & Non-responder \\
\hline
\end{tabular}

of treatment with interferon-alpha and ribavirin, and were followed-up for up to 6 months after medication. Serum collections were made at 12 and 24 weeks during treatment, and 7, 14, 21 and 28 days after the treatment had been completed, then monthly for up to 6 months. Coinfection with the human immunodeficiency virus (HIV) and/or with the hepatitis $B$ virus (HBV) was taken as an exclusion criterion. The project was approved by the research ethics committee of the São José do Rio Preto School of Medicine (FAMERP; opinion Nr. 087/2004).

\section{Molecular Modeling}

For the modeling of NS3, the restrain-based modeling approach was used as implemented in the MODELLER program [9]. A total of 1000 models were generated for each clone and the final model was selected based on objective function and stereochemical quality.

The stereochemical evaluation quality of the final model was assessed by PROCHECK [10], X-PLOR [11], Verify-3D [12] and WHATCHECK [13] programs.

\section{Molecular Docking Simulations}

The Molegro Virtual Docker program (version 2.0) was used to generate an ensemble of docked conformations for each variant helicase protein and ATP, RNA and Ribavirin compounds [14].

\section{Results and discussion}

Patients' characteristics

A total of 16 patients were treated and followed until 6 months after the end of the treatment. Seven patients (43.7\%) presented a virological sustained response
(VSR), of whom 6 were male and 1 was female. Nine patients $(56.3 \%)$ presented non-response (NR), 6 being male and 3 female. Thirteen patients were infected with $\mathrm{HCV}$ genotype $3 \mathrm{a}$ and 3 with $\mathrm{HCV}$ genotype $3 \mathrm{e}$. The patients' average age was 47.7 years (Table 1 ).

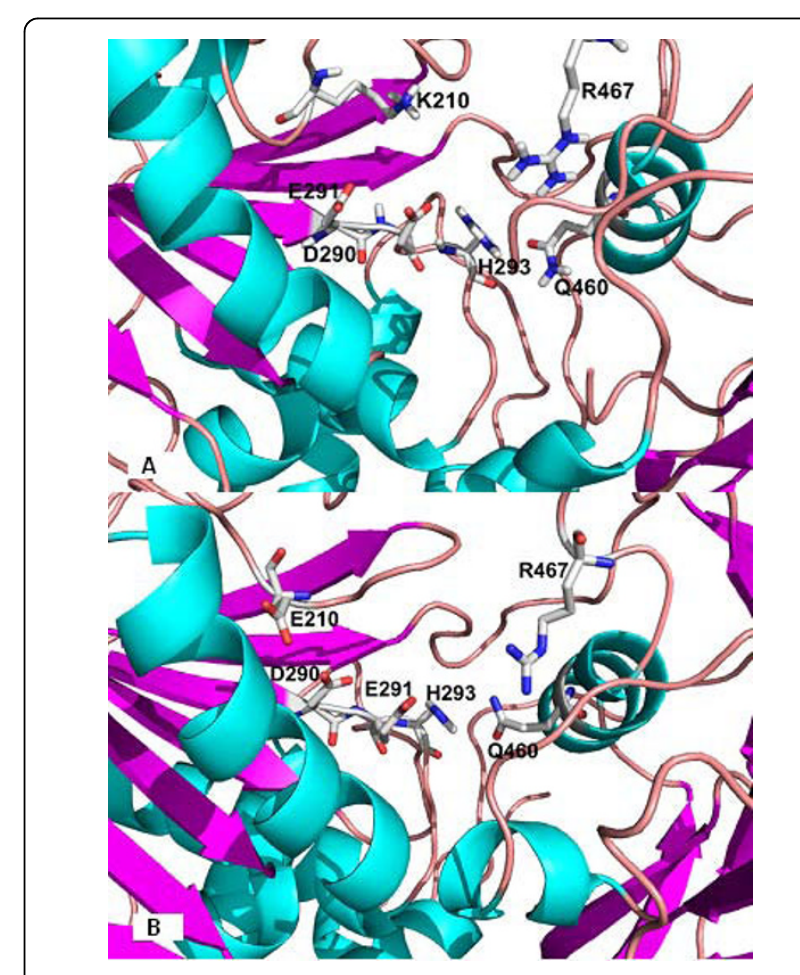

Figure 1 Key residues involved in ATP binding: (A) Template (PDB1A1V; Kim et al., 1998) [18], (B) patient RF007 showing the substitution of lysine for glutamic acid at position 210 of NS3. 


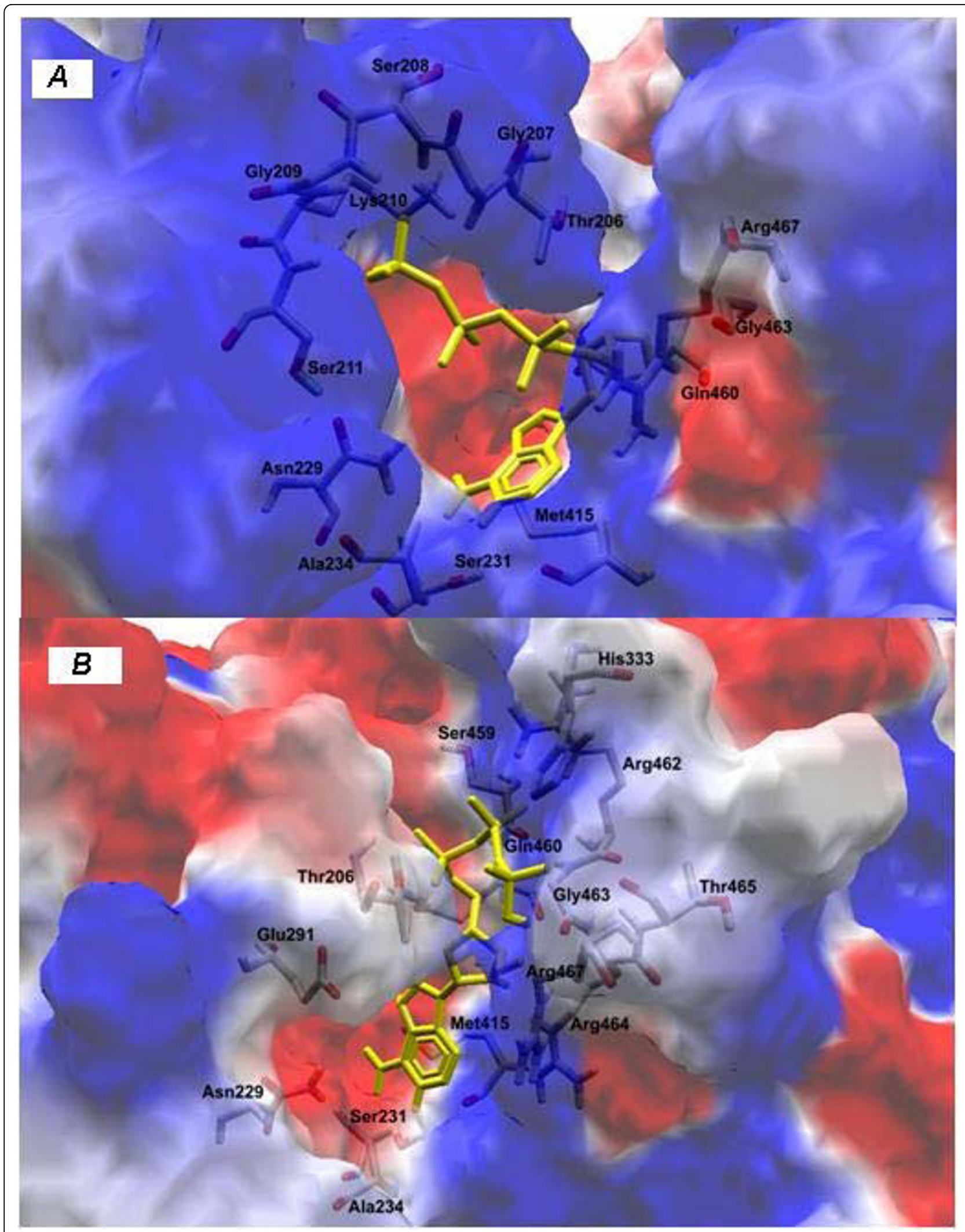

Figure 2 Docking simulations between helicase and ATP: (A) patient RF059 and (B) patient RF007 


\section{Analysis of the substitutions in the sequences NS3 Helicase}

Multiple sequence alignment analysis of the helicase fragment obtained from non-responder patients showed significant changes in helicase residues, for instance in the ATP and RNA binding sites. In patient RF007, a substitution of Lys210 for Glu210 occurred at the ATP binding site (Table 1). In the RNA binding site, patient RF020 had a Trp501 to Arg501 substitution (Table 1). No substitutions were found in the terminal region of the loop corresponding to a hydrophobic region (Phe531, Phe536 and Trp532). The arginine residue at position 393, important in the design of specific $\mathrm{HCV}$ NS3 inhibitors, was also assessed, but no changes were detected (Figure S1 of additional file 1).

PROTEUS showed variation in the number of $\alpha$-helix, beta-sheet and coil structures in all resistant patients (diagram I of additional file 1). The PROTPARAM analysis showed the substitutions in the protein's amino acids composition which leads to and compromises the structure as a whole in resistant patients (diagram II of additional file 1). However, since these are theoretical models, experimental techniques will be used to confirm the structural differences observed in the helicase portion, and to investigate whether there is a reduction of its affinity for the ligands. The elements of the tertiary structure showing that the substitutions subtly alter the quantitation of the secondary structure in Non-Responder patients due an increase in the random structure regions are shown in Figures S2A-S2C of additional file 1.

\section{Analysis of the helicase ATP-binding site}

Mutations in motifs I to VI located in the helicase domains 1 and 2 can impact on the ability of the protein to unwind genetic material and hydrolyze ATP, showing that these two activities are co-operative. Here, we have highlighted the K210E substitution (Table 1), which leads to a change of a positive to a negative charge in the altered residue (Figures $1 \mathrm{~A}$ and $1 \mathrm{~B}$ ), a different charge distribution and interactions between amino acids that might interfere with the hydrolysis of ATP.

To verify that the consequences the K210E substitution led to interaction with ATP, docking simulations were performed between helicase variant and the compost. The exchange of charges due to the substitution produced an imbalance in the distribution of loads, leading to a decrease in the number of ligations between the essential amino acids for the exercise of hydrolysis of ATP (Figures 2A-2B). ATP and a required metal ion cofactor (i.e. $\mathrm{Mg}^{+}$) normally bind to a helicase in the cleft that separates two adjacent RecA-like domains. The most critical residues for ATP binding arise from the Walker A and B motifs. The Walker A motif of $\mathrm{HCV}$ helicase forms a phosphate binding loop (P-loop) with the conserved Lys210, thereby probably contacting the $\gamma$ phosphate of ATP [15]. With this disturbance, substitution of Lys 210 caused a mild disruption of the molecule accompanied by a change in its orientation.

Kim et al. [16] through site-directed mutations showed that the K210E mutant lost its RNA helicase activity and had very little NTPase activity compared to the wild-type protein. Similar results were observed by Chang et al. [17], in which the ATPase activity was significantly reduced in mutant NS3 proteins (K210E). They also observed that the wild type NS3 protein completely unwound the RNA substrate in the presence of ATP, but the mutation (K210E) completely abrogated the activity.

\section{Analysis of the helicase RNA-binding site}

The substitution (W501R; see Table 1) of a non-polar (neutral) amino acid for a polar (positive) residue (Figures 3A and 3B) leads to an increase of positivity in this region and thus alters the interaction of the protein with its environment. Docking simulations between helicase variant and the RNA substract

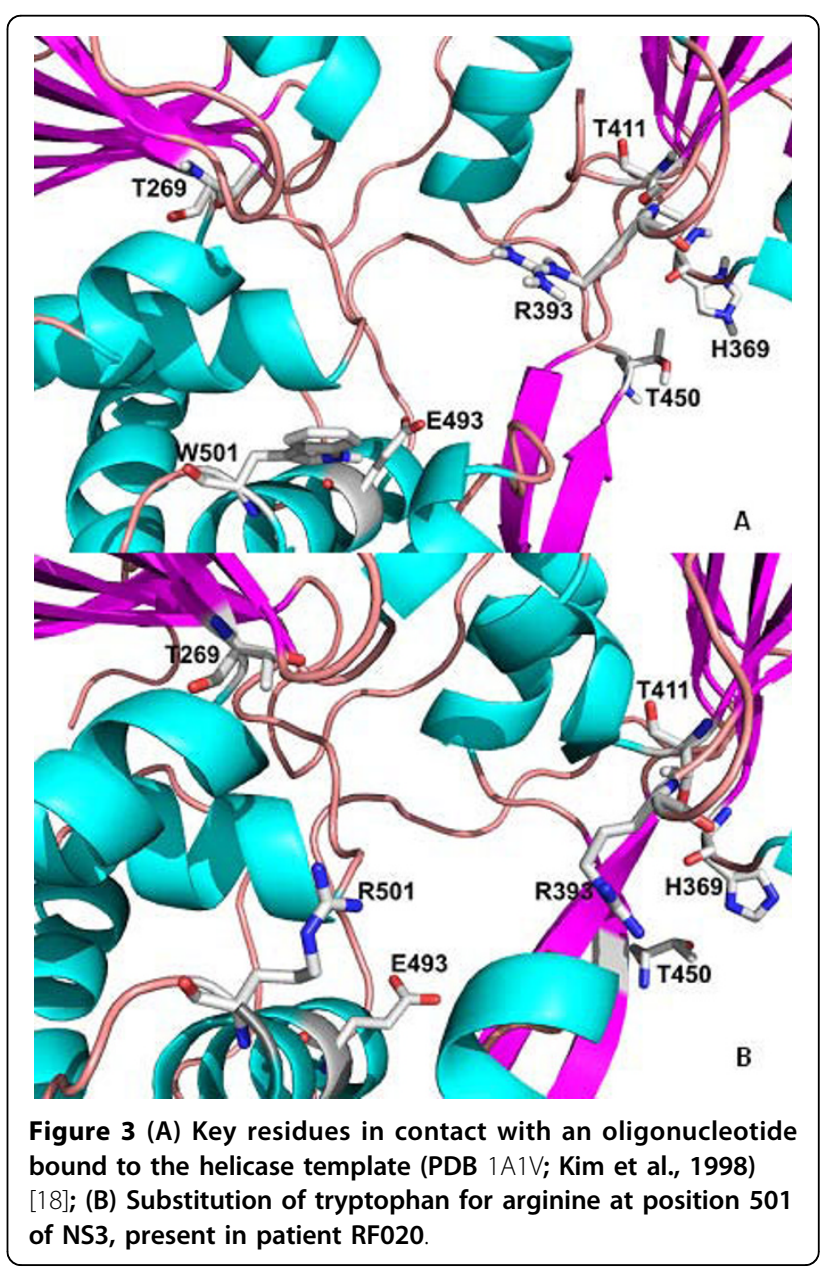




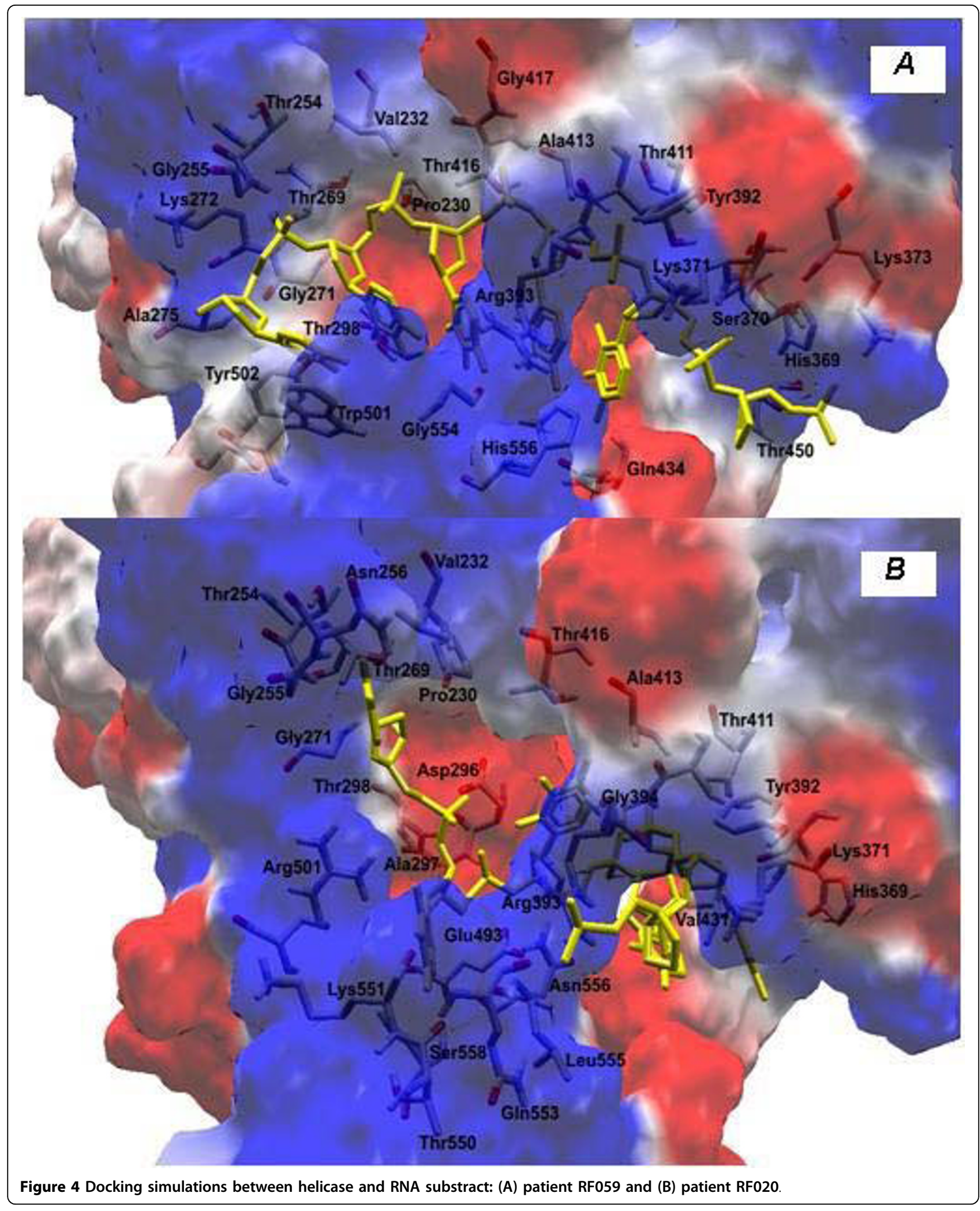




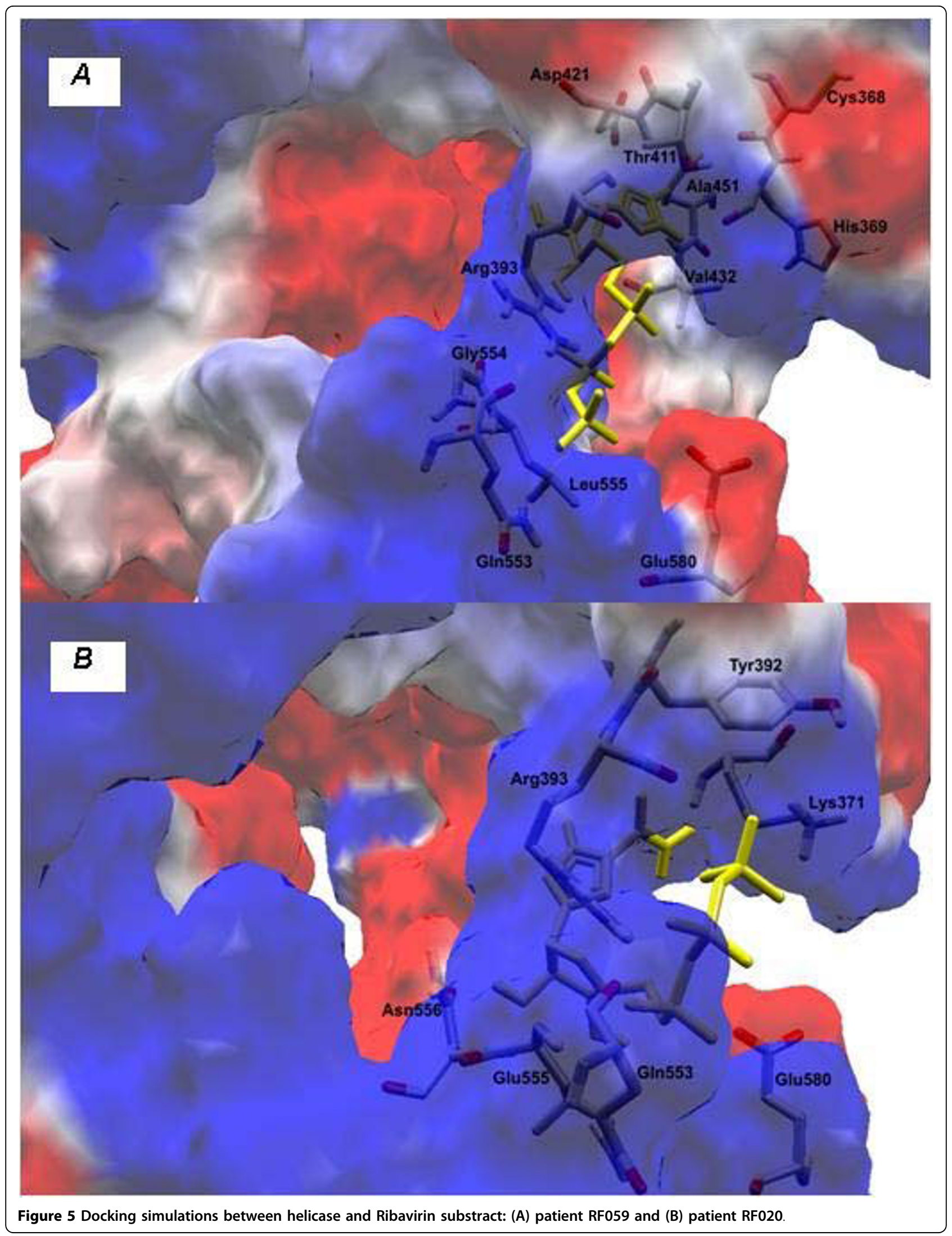


showed that the substitution of tryptophan by arginine at position 501 causes an imbalance in the distribution of loads leading, and forces the RNA to interact with the amino acid, Thr269. In this case, we observed an increase in the positive charges near to R501 (Figures $4 \mathrm{~A}$ and $4 \mathrm{~B})$.

Based on the observation that the oligonucleotide appears to be locked in the binding cleft because a residue in domain 3, Trp501, is stacked against the 3'-terminal base, Kim et al. [18], proposed that ATP binding, and the subsequent closure of the cleft between domains 1 and 2, will lead to a ratcheting of Trp501 past 1 or 2 nucleotides. Consequently, the protein would move towards the 5 '-end of the bound nucleic acid. After ATP is hydrolyzed and Trp501 is once more locked into a place acting as a bookend, the cleft opens and RNA slides through to the other side of the protein. Consequently the replacement of W501 prevents the correct movement of the protein in the "ratcheting inchworm" model first proposed for HCV helicase by Kim et al. [18].

Since interferon-alpha did not act as a protease inhibitor, we also performed docking simulations between the variant protein W501R and the compound Ribavirin to find a correlation between the substitutions found in Helicase and the response of patients to treatment. This inhibitor connects to altered Helicase the same way that as in Helicase wild type, independent of the substitution of tryptophan by arginine at position 501, and thereby maintaining its function (Figures 5A-5B). From this, we infer that the mutation does not prevent the binding of inhibitor, but indicates that this is not the reason for the lack of response to treatment.

\section{Conclusions}

Natural occurrence of amino acids substitution in nonresponder $\mathrm{HCV}$ patients has been explored for important sites in relation to the perfect excise of helicase function. Correlations of the variants L210E and W501R with patients' responses to the treatment may not be valid at this time because other forms of viruses that have the helicase in their wild types are predominant in the patient, thus ensuring viral replication. It is important to find these variants so that future enzymatic studies can be carried out on them. Individual analysis of treatment with future inhibitors can be important to treatment response.

\section{Additional material}

Additional file 1: Methods, Figure S1 and Figure S2Methods, Figure S1 and S2

\section{Acknowledgements}

This work was supported by grants from FAPESP, CNPq and CAPES.

\section{Author details}

'São Paulo State University - UNESP, Department of Biology, São José do Rio Preto/SP, CEP: 15054-000, Brazil. 'Faculty of Medicine, University of São Paulo - USP, Department of Medical Clinic, São Paulo/SP, CEP: 01246-903, Brazil.

${ }^{3}$ Butantan Institute, Viral Immunology Laboratory, São Paulo/SP, Brazil. ${ }^{4}$ Faculty of Medicine, University of São Paulo - USP, Department of Gastroenterology, São Paulo/SP, CEP: 05503-900, Brazil. ${ }^{5}$ Faculty of Medicine of São José do Rio Preto, São José do Rio Preto, CEP: 15090-000, Brazil. 'São Paulo State University - UNESP, Center of Study of Social Insects/Department of Biology, Rio Claro/SP, CEP: 13506-900, Brazil.

\section{Authors' contributions}

PJSP carried out the experiments, acquisition of data, analysis and interpretation of data and drafting the manuscript; HAA and MSP carried out the molecular modeling and suggestions in this manuscript; IMVGCM participated in the study and made suggestions to the manuscript; JRRP and MLN participated in the study; PR conceived the study, participated in its analysis and coordination and suggestions in this manuscript. All authors read and approved the final manuscript.

\section{Competing interests}

The authors declare that they have no competing interests.

Received: 2 June 2010 Accepted: 14 July 2010 Published: 14 July 2010

References

1. Lauer GM, Walker BD: Hepatitis C virus infection. N Engl J Med 2001, 345:41-52.

2. World Health Organization: [http://www.who.int/en/]

3. Interagency Network of Information for Health: [http://www.ripsa.org.br/ fichasIDB/record.php?lang=pt\&node=D.1.14].

4. Choo QL, Kuo G, Weiner AJ, Overby LR, Bradley DW, Houghton M: Isolation of a cDNA clone derived from a blood-borne non- $A$, non-B viral hepatitis genome. Science 1989, 244:359-362.

5. Kato N, Hijikata M, Ootsuyama Y, Nakagawa M, Ohkoshi S, Sugimura T, Shimotohno K: Molecular cloning of the human hepatitis $C$ virus genome from Japanese patients with non-A, non-B hepatitis. Proc Natl Acad SCi USA 1990, 87:9524-9528.

6. Takamizawa A, Mori C, Fuke I, Manabe S, Murakami S, Fujita J, Onishi E, Andoh T, Yoshida I, Okayama H: Structure and organization of the hepatitis C virus genome isolated from human carriers. J Virol 1991 65:1105-1113.

7. Hijikata M, Mizushima H, Akagi T, Mori S, Kakiuchi N, Kato N, Tanaka T, Kimura K, Shimotohno K: Two distinct proteinase activities required for the processing of a putative nonstructural precursor protein of hepatitis C virus. J Virol 1993, 67:4665-4675.

8. Frick DN: The hepatitis C virus NS3 protein: a model RNA helicase and potential drug target. Curr Issues Mol Biol 2007, 9:1-20.

9. Sali A, Blundell TL: Comparative protein modelling by satisfaction of spatial restraints. J Mol Biol 1993, 234:779-815.

10. Laskowski RA, MacArthurm MW, Smith DK, Jones DT, Hutchinson EG, Morris AL, Naylor D, Moss DS, Thornton JM: PROCHECK v.3.0 - Program to check the stereochemistry quality of protein structures - Operating instructions. 1994.

11. Brünger AT: In: X-PLOR, A System for Crystallography and NMR. Yale Univ Press New Haven CT 1992, Version 3.1.

12. Luthy $R$, Bowie $J U$, Eisenberg D: Assessment of protein models with three-dimensional profiles. Nature 1992, 356:383-385.

13. Vriend G: WHAT IF: a molecular modeling and drug design program. J Mol 1990, 8:52-56.

14. Thomsen Rene, Christensen Mikael H: MolDock: A New Technique for High-Accuracy Molecular Docking. J Med Chem 2006, 49:3315-3321.

15. Walker JE, Saraste M, Runswick MJ, Gay NJ: Distantly related sequences in the alpha- and beta-subunits of ATP synthase myosin, kinases and other ATP-requiring enzymes and a common nucleotide binding fold. EMBO J 1982, 1:945-951. 
16. Kim DW, Kim J, Gwack Y, Han JH, Choe J: Mutational analysis of the hepatitis C virus RNA helicase. J Virol 1997, 71:9400-9.

17. Chang SC, Cheng JC, Kou YH, Kao CH, Chiu CH, Wu HY, Chang MF: Roles of the AX(4)GKS and arginine-rich motifs of hepatitis $C$ virus RNA helicase in ATP- and viral RNA-binding activity. J Virol 2000, 74:9732-7.

18. Kim JL, Morgenstern KA, Griffith JP, Dwyer MD, Thomson JA, Murcko MA, Lin C, Caron PR: Hepatitis C virus NS3 RNA helicase domain with a bound oligonucleotide: the crystal structure provides insights into the mode of unwinding. Structure 1998, 6:89-100.

doi:10.1186/1756-0500-3-196

Cite this article as: Provazzi et al: Structural studies of Helicase NS3 variants from Hepatitis $C$ virus genotype 3 in virological sustained responder and non-responder patients. BMC Research Notes 2010 3:196.

\section{Submit your next manuscript to BioMed Central} and take full advantage of:

- Convenient online submission

- Thorough peer review

- No space constraints or color figure charges

- Immediate publication on acceptance

- Inclusion in PubMed, CAS, Scopus and Google Scholar

- Research which is freely available for redistribution

Submit your manuscript at www.biomedcentral.com/submit 